\title{
Hybrid Angular- and Energy-Dispersive X-ray Diffraction Computed Tomography
}

\author{
Robert M. Moss, Matthew D. Wilson, Edward J. Morton and Robert D. Speller
}

\begin{abstract}
X-ray diffraction is a material-specific technique, the results of which can be used as a material fingerprint to identify unknowns. In this paper we present an adaptation to a novel hybrid angular- and energy-dispersive $X$-ray diffraction technique, which, until now, has been limited in utility by sample thickness. Computed tomography techniques have been applied to spatially resolve the origin of the scattering signals and to reconstruct the diffraction pattern in each pixel. 2D crosscorrelation has been used to compare the reconstructed data to a library of standards as a means of identifying the material present.
\end{abstract}

\section{INTRODUCTION}

$\mathrm{X}_{\mathrm{b} e \mathrm{~s}}^{\mathrm{n}}$ -RAY diffraction (XRD) is a powerful technique which can be used to probe the structure of matter. When X-rays interact with a material they can be scattered elastically (no change in energy) and coherently (no change in phase) and there is a possibility of wave-like interference occurring. The traditional approach to XRD is angular-dispersive, where a beam of monochromatic X-rays (having fixed wavelength, $\lambda$ ) is incident upon the sample and the scattering intensity is measured as a function of scattering angle $(2 \theta)$ around the sample. The fixed and repeating atomic arrangement of a crystalline material form planes separated by a consistent distance $\left(d_{n}\right)$ which allows the scattered X-rays to constructively interfere where Braggs Law (1) is satisfied. An alternative approach is energy-dispersive XRD where a polychromatic beam of X-rays is used and an energy resolving detector is positioned to measure the scattering intensity at a fixed scattering angle. Since each material has a unique atomic structure, the scattering pattern can be used as a fingerprint to identify unknowns.

$$
\lambda=2 d_{n} \sin \theta
$$

A hybrid approach to XRD combines angular- and energydispersive techniques [1]. Key to this method is HEXITEC: a cutting edge multi-element (pixelated) energy-dispersive detector which is capable of providing a full energy histogram in every pixel [2]. Usually, knowledge of the scattering geometry is used to assign a scattering angle to every pixel such that the energy histograms can be represented in momentum transfer $(x)$ space via (2), where the denominator is the product of Planck's constant $(h)$ and the speed of light $(c)$.

$$
x=(E \sin \theta) / h c
$$

This allows the data from all pixels to be summed together which increases collection efficiency. However, if the sample

Manuscript received December 7, 2018. This work was supported the Science and Technology Facilities Council (STFC) Innovations Partnership Scheme (IPS) under Grant ST/P000088/1 and Rapiscan Systems.

R. M. Moss and R. D. Speller are with the Department of Medical Physics and Biomedical Engineering, University College London, Gower Street, London WC1E6BT (email: robert.moss@ucl.ac.uk). is thick (more than a few $\mathrm{mm}$ ) then the range of scattering angles each pixel can 'see' becomes broader and the diffraction pattern loses resolution and specificity. Therefore, techniques need to be developed which allow greater spatial separation of the scattering locations for thick samples while maintaining the advantages of the hybrid methodology.

\section{METHOD}

The experimental setup is shown in Fig. 1. A compact X-ray generator (Source-Ray SB-80-1K) was operated at $80 \mathrm{kV}$ and 1 $\mathrm{mA}$ to provide a polychromatic X-ray spectrum. A pair of collimators with a $1 \mathrm{~mm}$ diameter pinhole were used to define a pencil beam. The sample was mounted on translation and rotation stages. A small lead beam stop was used to block the primary beam after the sample. The HEXITEC detector was position to record X-rays scattered from the region where the primary beam intersected the sample. Photon counts were assigned to energy histogram bins of $0.25 \mathrm{keV}$. Data were collected for 35 lateral increments at $1 \mathrm{~mm}$ intervals and 36 rotation increments at $10^{\circ}$ intervals for $10 \mathrm{~s}$ at each position. The position of the primary beam with respect to the detector pixels was determined and the detector counts were integrated radially to create a $4 \mathrm{D}$ matrix having dimensions of photon energy $(E)$, radial distance from the beam centre $(r)$, sample rotation angle $(\varphi)$ and lateral sample position $(S)$.

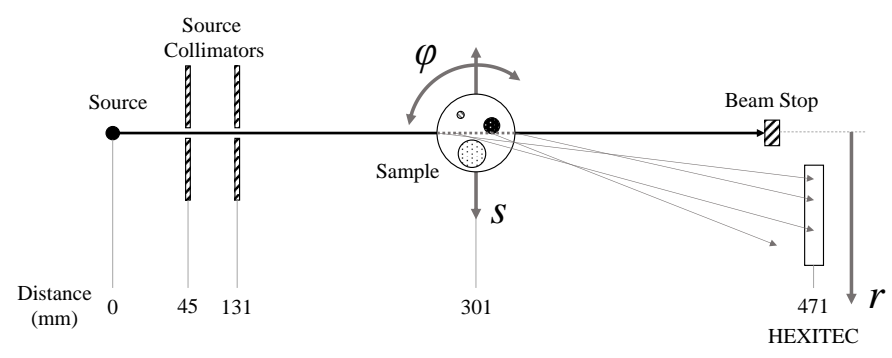

Fig. 1: Schematic diagram of the experimental setup used to collect the XRD projections.

A test phantom was constructed with three cylindrical scattering components: $4.5 \mathrm{~mm}$ caffeine powder (packed in a drinking straw), $6.4 \mathrm{~mm}$ polytetrafluoroethylene (PTFE) rod and $16 \mathrm{SWG}(\sim 1.6 \mathrm{~mm})$ aluminium wire. Sinograms were extracted by taking slices along the $(\varphi, S)$ plane of the $4 \mathrm{D}$

M. D. Wilson is with the Detector Development Group, STFC Rutherford Appleton Laboratory, Harwell Campus, Didcot OX11 0QX, UK

E. J. Morton is with Rapiscan Systems, 2805 Columbia Street, Torrance, CA, 90503, USA 
matrix and conventional filtered back-projection (shown to be effective for this problem [3]-[5]) was used to reconstruct diffraction images for every combination of $E$ and $r$.

XRD data were also collected for a series of sample materials to create a limited reference library. The samples were placed at the mean sample-to-detector distance and the XRD pattern was recorded for each using the conventional hybrid approach [1]. The library contained caffeine, aluminium plate, PTFE and Mylar film, as described in a previous study [6], Lanex screen, aluminium wire and air (i.e. no sample present).

\section{RESULTS AND DISCUSSION}

The reconstructed images were $35 \times 35$ pixels (defined by the sampling rate of $\varphi$ and $S$ ) which show three locations of elevated scattering as expected. Fig. 2 shows the sinograms and the reconstructed images for the global (total) scattering intensity as well as for three different $x$ values using (2). In this case $\theta$ has been calculated via (3) using a single value of $r$ $(17.25 \mathrm{~mm})$ and the mean sample-to-detector distance $\left(D_{s d}\right)$ of $170 \mathrm{~mm}$. It can be seen that the different components appear more or less clearly depending on the $x$ window being observed.

$$
\tan 2 \theta=r / D_{s d}
$$
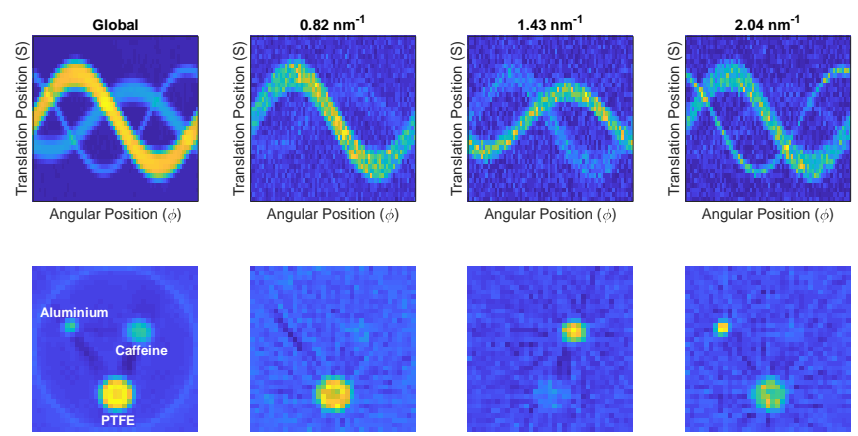

Fig. 2: Summed (global) scattering image showing the locations of the phantom components and pixel intensities as a function of $x$ showing how contrast can be achieved between different materials.

From the reconstruction it is possible to extract the scattering intensity as a function of $E$ and $r$, so called ' $(E, r)$ maps', for each pixel. Fig. 3 shows the $(E, r)$ maps for aluminium, PTFE and caffeine from the reference library and the appropriate reconstructed pixel respectively. In all images it is possible to observe several bright arcs which correspond to lines of constant $x$.

Normalised 2D cross-correlation analysis [7] was used to compare the reconstructed $(E, r)$ map in each pixel to the library of standards. Fig. 4 shows the classification of pixels where the colours indicate different material assignments. It can be seen that there is generally good agreement between the spatial classification of the pixels and the physical layout of the phantom. Cross-correlation analysis outputs values $\leq 1$, where 1 indicates a perfect match. As such, this can be used as a crude measure of 'confidence' in the assigned classification which is shown pictorially in Fig. 4. The classification confidence is high (close to 1) where the major contribution to the pixel come from a single material and low where mixture/boundaries exist.

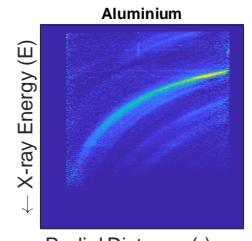

Radial Distance $(r) \rightarrow$

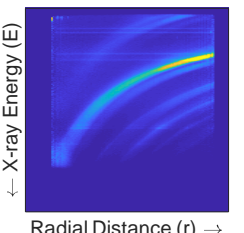

Radial Distance $(r) \rightarrow$
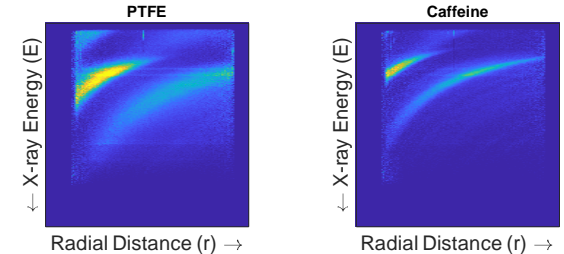

Radial Distance $(r) \rightarrow$
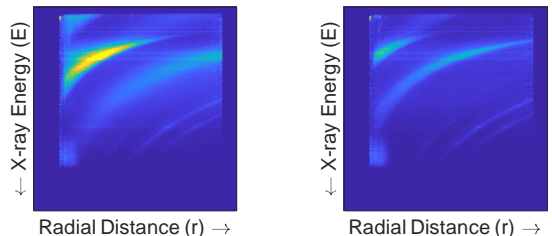

Fig. 3: $(E, r)$ maps for aluminium, PTFE and caffeine comparing the standards measured in isolation (lower row) and the reconstructed pixels (upper row). The horizontal axis is $r$ in steps of $0.25 \mathrm{~mm}$ and the vertical axis is $E$ is steps of $0.25 \mathrm{keV}$.
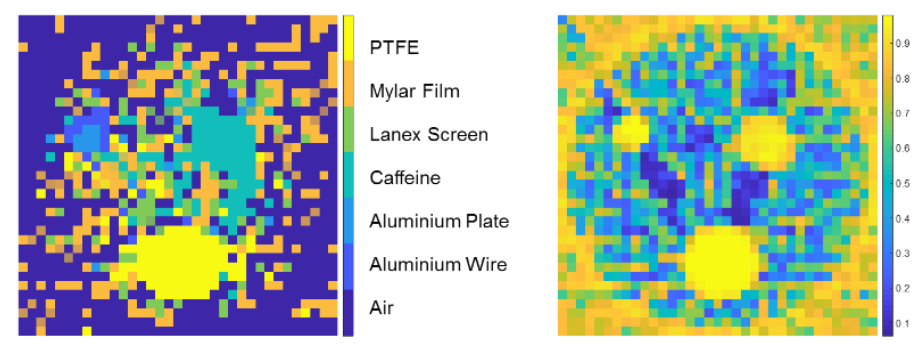

Fig. 4: Pixel material classification based on 2D cross-correlation analysis (left) and 'confidence' of the classification in each pixel (right).

It should be noted that air and Mylar film are approximately the same because the scattering contribution from Mylar is negligible. It can be seen that the material assignment for pixels between the three scattering objects in the phantom is occasionally wrong (e.g. Lanex screen was not present in the phantom but has been assigned to some pixels) but this is coupled with a reduced confidence of assignment in this region.

\section{CONCLUSIONS}

We have successfully demonstrated a hybrid angular- and energy-dispersive X-ray diffraction system for 3D material discrimination using computed tomography techniques. We have been able to separate the scattering contributions of three distinct materials from each other and from the background. Normalised 2D cross-correlation provided a simple method for material classification based on comparing reconstructed $(E, r)$ maps with those collected for standard materials. The agreement between the pixel classification and the physical layout of the phantom is good.

\section{REFERENCES}

[1] D. O'Flynn et al., 'Explosive detection using pixellated X-ray diffraction (PixD)', J. Instrum., vol. 8, no. 03, p. P03007, 2013.

[2] P. Seller et al., 'Pixellated $\mathrm{Cd}(\mathrm{Zn}) \mathrm{Te}$ high-energy X-ray instrument', $J$. Instrum., vol. 6, no. 12, p. C12009, 2011.

[3] Z. Zhu, A. Katsevich, A. J. Kapadia, J. A. Greenberg, and S. Pang, 'X-ray diffraction tomography with limited projection information', Sci. Rep., vol. 8, no. 1, p. 522, Jan. 2018. 
[4] G. Harding and J. Kosanetzky, 'Elastic scatter computed tomography', Phys. Med. Biol., vol. 30, no. 2, p. 183, 1985.

[5] J. A. Griffiths, G. J. Royle, J. A. Horrocks, A. M. Hanby, and R. D. Speller, 'Correlation of Diffraction MicroCT Images of Breast Tissue with Pathological Analysis', in IEEE Nuclear Science Symposium Conference Record, 2006, 2006, vol. 5, pp. 2936-2939.

[6] R. Moss, C. Crews, M. Wilson, and R. Speller, 'miniPixD : a compact sample analysis system which combines X-ray imaging and diffraction', J. Instrum., vol. 12, no. 02, p. P02001, 2017.

[7] J. P. Lewis, Fast Normalized Cross-Correlation. 1995. 